Check for updates

Cite this: RSC Adv., 2019, 9, 39332

\title{
A mechanochemically synthesized porous organic polymer derived CQD/chitosan-graphene composite film electrode for electrochemiluminescence determination of dopamine $\uparrow$
}

\author{
Qianxiu Pan, ${ }^{\text {a }}$ Zhilu Xu, ${ }^{\text {ab }}$ Shue Deng, ${ }^{a}$ Fenglian Zhang, ${ }^{a}$ Hui Li, ${ }^{a}$ Yuanzheng Cheng, ${ }^{a}$ \\ Liuya Wei, ${ }^{a}$ Jiangyun Wang ${ }^{a}$ and Baolong Zhou (D) *ab
}

\begin{abstract}
Herein, we explore a new carbon source for preparation of carbon quantum dots (CQDs) with controllable composition using a porous organic polymer (POP) derived porous carbon via a nitric acid oxidation method. The POP used for the preparation of CQDs was synthesized by mechanochemical FriedelCrafts alkylation under solvent free conditions. Using the as-prepared CQDs, we develop a simple and effective electrochemiluminescence (ECL) detection method for dopamine (DA) using a CQD/chitosangraphene composite modified glassy carbon electrode (GCE). Both the electrochemical and ECL behaviors were studied in detail with ammonium persulfate as a coreactant. The complementary structure and synergistic function of the composite give the ECL sensor special properties. Apart from the high stability, it also presents good repeatability and high sensitivity to DA with a wide linear range from 0.06 to $1.6 \mu \mathrm{M}$. And a satisfactory detection limit of $0.028 \mu \mathrm{M}(\mathrm{S} / \mathrm{N}=3)$ was achieved for the prepared sensor. Furthermore, the ECL also shows high selectivity toward DA with an excellent interference resistance ability at a high concentration ratio of $100\left(C_{\text {interference }}: C_{\mathrm{DA}}=100\right)$. In addition, the ECL sensor was successfully applied for effective detection and quantitative analysis of the actual dopamine in human body fluids for disease diagnosis and pathological studies.
\end{abstract}

Received 2nd September 2019 Accepted 18th November 2019

DOI: $10.1039 / \mathrm{c} 9 \mathrm{ra06912g}$

rsc.li/rsc-advances extensively studied. EL is electrically driven emission of light from the fluorescent materials..$^{12}$ Numerous EL materials have been developed and considerable research interest have been geared. ${ }^{13-17}$

Carbon quantum dots (CQDs), as a new nanomaterial with excellent stability, well biological compatibility and good aqueous solubility, are regarded as new candidates for luminescent materials. ${ }^{18-22}$ It have been anticipated to overtake previous pioneers in many fields. ${ }^{23}$ Up to now, various carbon source had been used for the preparation of CQDs, i.e., activated carbon, carbon nanotubes (CNTs), biomass, organic molecular and even metal organic frameworks (MOFs), but, preparing CQDs with controllable element composition remains a huge challenge. ${ }^{24}$ It is desirable to explore a low-cost and eco-friendly precursor for controllable preparation of CQDs. ${ }^{25}$

Porous organic polymers (POPs) are multifunctional materials composed by pure organic units via covalent bond. ${ }^{26}$ The modular construction principle made the structure and composition deterministic at atom level. Recent reports have evidenced it an ideal candidates for accurate preparation of carbonous materials with definite composition. Hence, it is also a promising material for target synthesis of CQDs. ${ }^{27,28}$ In this article, CQDs was prepared from a POP (TPE-HCP) derived 
porous carbon (TPE-800) via the typical acidic oxidation method. TPE-HCP was prepared via the mechanochemical Friedel-Crafts alkylation reaction under the catalytic of $\mathrm{FeCl}_{3}$ in solvent free conditions. ${ }^{29}$

Chitosan is an amino and hydroxyl rich polysaccharide with good biocompatibility. Furthermore, as a linear polymer, it also presented excellent film-forming property which was extensively applied in modified electrode. ${ }^{17}$ Graphene is a two-dimensional (2D) material with high BET surface areas, super electrical conductivity and good film-processing ability. ${ }^{18}$ The inherent property make it easy to compound with other materials. Bear that in mind, a novel chitosan-graphene (CG) composite combining the characteristic of graphene and chitosan were developed. The composite possessed large amount of amino and hydroxyl which is more conducive to carrying CQDs.

In this article, we presented a simple but effective ECL method for detection of DA based on an original electrode modified by CQDs/CG composite film. The CQDs/CG electrode showed a high selectivity to DA with a wide linear range (0.06 to $1.6 \mu \mathrm{M})$ and a satisfying detection limit $(0.028 \mu \mathrm{M}, \mathrm{S} / \mathrm{N}=3)$. And the composite electrode also presented an excellent interference resistance ability towards both the organic (glucose, ascorbic acid and uric acid) and inorganic $\left(\mathrm{Na}^{+}, \mathrm{K}^{+}\right)$interfering substance. Furthermore, it also exhibited excellent stability, good reproducibility and high sensitivity to DA. Furthermore, the prepared sensor could be applied for detection of DA in real samples.

\section{Results and discussion}

As shown in Fig. 1, TPE-HCP was prepared via the mechanochemical Friedel-Crafts alkylation reaction with the catalytic of $\mathrm{FeCl}_{3}$ in a planet ball mill under solvent free conditions. In brief, to a $50 \mathrm{~mL}$ zirconium oxide milling vessel, 1,3,5-triformylbenzene (TBM, $713.8 \mathrm{mg}, 1.5 \mathrm{mmol}$ ), 1,1,2,2-tetraphenylethene (TPE, $498.7 \mathrm{mg}, 2 \mathrm{mmol}$ ) and anhydrous iron(III) chloride (2.92 g, $18 \mathrm{mmol}$ ) were added. Consequently, the vessel was milled at $500 \mathrm{rpm}$ for 35 minutes. After, the resulting polymers were washed with hydrochloric acid solution (1 M $\mathrm{HCl})$ and methanol to remove the catalysts adequately. Then the

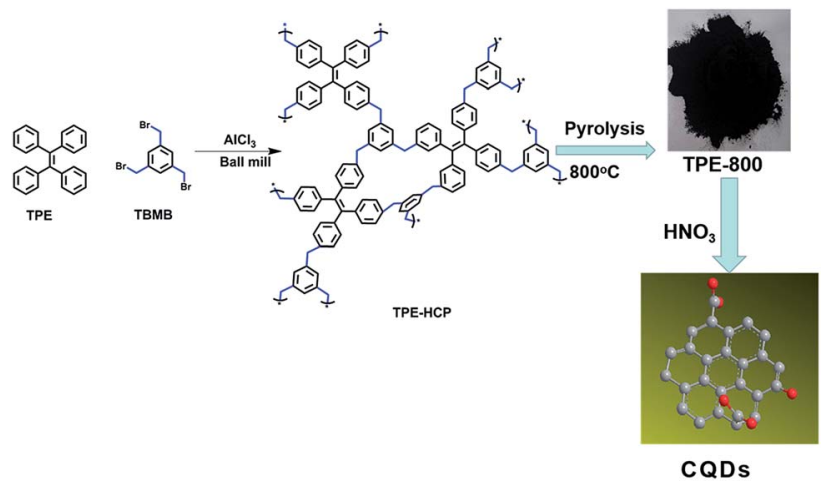

Fig. 1 The typical route for the preparation of TPE-HCP and corresponding porous carbon (TPE-800) together with the synthesis of CQDs. (Red atom is oxygen and the grey is carbon). obtained solid was dried at a vacuum oven at $80{ }^{\circ} \mathrm{C}$ for 12 hours. And the corresponding porous carbon (TPE-HCP-800) were obtained via direct carbonization of as-synthesized polymer under Ar at $800{ }^{\circ} \mathrm{C}$. The CQDs were synthesized through the $\mathrm{HNO}_{3}$ acid oxidation of TPE-800. The detail was given in the ESI. $\dagger$

The construction of porous skeletons and the formation of CQDs was initially validated by Fourier infrared spectrometer (FTIR). As shown in Fig. S1a, $\uparrow$ the bromine methyl vibration (C$\mathrm{Br}$ ) at $680 \mathrm{~cm}^{-1}$ has completely vanished and typical vibrations assignable to the aliphatic connections appeared. ${ }^{\mathbf{1 6}}$ Both proved the formation of polymer skeleton. The CQDs used for the FTIR tests was obtained by rotary evaporators at $70{ }^{\circ} \mathrm{C}$. Fig. S1c $\dagger$ presents the FTIR spectrum of as-prepared CQDs. Different with the spectrum of TPE-800 (Fig. S1b $\dagger$ ), a wide peak around $3324 \mathrm{~cm}^{-1}$ assigned to the $\mathrm{O}-\mathrm{H}$ stretching vibration could be clearly observed. Furthermore, the peak located at $1334 \mathrm{~cm}^{-1}$ attributed to the $\mathrm{C}-\mathrm{OH}$ vibration could also be detected. Other characteristic absorption signals at $1574 \mathrm{~cm}^{-1}$ and $1423 \mathrm{~cm}^{-1}$ are assigned to the vibration of $\mathrm{C}=\mathrm{O}$ and the $-\mathrm{COO}-$ in $-\mathrm{COOH}$ groups. ${ }^{30}$ Therefore, the as-synthesized CQDs are rich in $\mathrm{C}-\mathrm{OH}$ and $\mathrm{COOH}$ groups. Solid-state ${ }^{13} \mathrm{C}$ cross polarization magic angle spinning (CP/MAS) NMR were also performed to investigate the covalent linkage of the building units of prepared porous polymers. As exhibited in Fig. S1d, $\uparrow$ resonance peaks near $35 \mathrm{ppm}$ ascribed to the methylene could be clearly detected, indicating the formation of methylene linker in the polymer networks after the mechanochemical Friedel-Crafts reaction. In addition, UV absorption and fluorescence spectra were also conducted to testify the formation of CQDs. As shown in Fig. S2, $\dagger$ a obvious absorption peak at $229 \mathrm{~nm}$ belonging to the characteristic peak of CQDs could be observed. And the fluorescence emission peak (excited at the wavelength of 400 $\mathrm{nm}$ ) appeared at $567 \mathrm{~nm}$.

Low temperature $\mathrm{N}_{2}$ uptake measurements and pore width distribution curves (Fig. S3a and $\mathrm{b}_{\dagger} \dagger$ ) testified the inherent porosity of synthesized polymer and corresponding porous carbon. The detail was listed in the Table $\mathrm{S} 1 . \dagger$ Like the reported works, almost no clear peaks could be detected form the powder XRD (Fig. S4a $\dagger$ ), proving the amorphous structure of assynthesized samples. TG curve are given in the Fig. S3c, $\dagger$ one could observe clearly that the weight could keep at $60 \%$ even at the temperature of $800{ }^{\circ} \mathrm{C}$, implying excellent thermal stability of prepared polymer. Raman spectrum (Fig. $\mathrm{S} 4 \mathrm{~b} \dagger$ ) validated the high degree of graphitization of TPE-800 with a intensity ratio of $0.948\left(I_{\mathrm{D}}: I_{\mathrm{G}}=0.948\right)$. However, the target CQDs shows a intensity ratio of 0.991 . Furthermore, XPS was performed to investigate the element composition of prepared porous carbon (Fig. S5 $\dagger$ ). The survey spectrum identified the coexistence of $\mathrm{C}$ and $\mathrm{O}$ in the porous skeletons (Fig. S5a $\dagger$ ).

In order to investigate the morphology of prepared materials, scanning electron microscope (SEM) and high-resolution transmission electron microscope (HR-TEM) were performed (Fig. 2). Fig. 2a and b present the SEM of synthesized polymer and corresponding porous carbon in a scale bar of $500 \mathrm{~nm}$. Intergrown spherical particles stacked in a bulk could be clearly observed (Fig. 2a). Just like the morphology of polymer, bulk stacked by spherical particles could be detected from the SEM of 

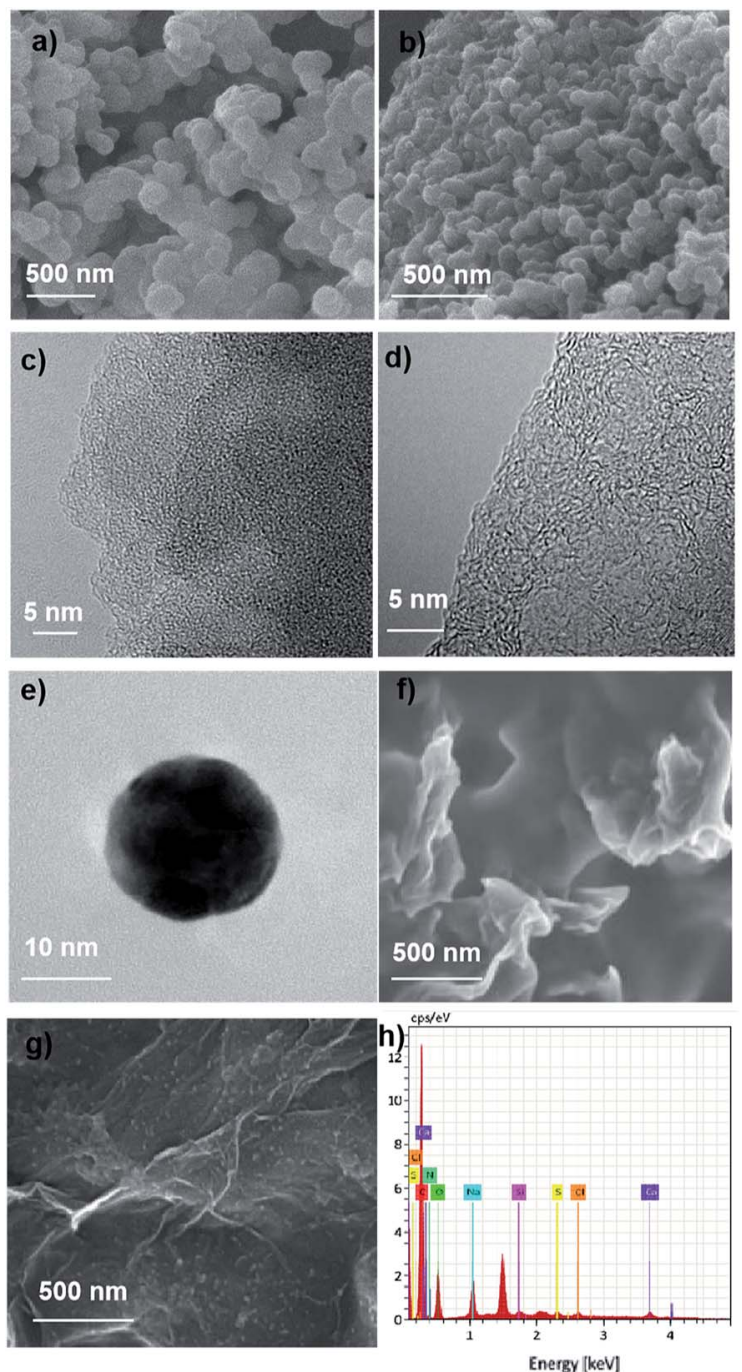

Fig. 2 SEM and TEM of prepared samples: (a) SEM of TPE-HCP; (b) SEM of TPE-800; (c) high-resolution TEM of TPE-HCP; (d) highresolution TEM of TPE-800; (e) TEM of single CQDs; (f) SEM of chitosan-graphene composite film; (g) SEM of CQDs/chitosan-graphene composite film; (h) EDS of a single CQD obtained form the high resolution TEM.

TPE-800 (Fig. 2b). High resolution TEM of TPE-HCP and TPE800 are delivered in Fig. 2c and d, respectively, from which, widely distributed micropore could be found. Different with the polymer precursor, obvious graphitic stripe could be detected from Fig. 2d. Fig. 2e exhibits a single CQD taken by casting the newly acquired CQDs solution. A regular spherical shape with a diameter of about $15 \mathrm{~nm}$ could be observed. Fig. 2f exhibits the SEM image of the chitosan-graphene membrane. A rough and large surface could be detected. The immobilization of CQDs on the surface of chitosan-graphene membrane film could be validated by SEM image of the CQDs/CG composite film. Large amounts of CQDs distributed homogeneously on the surface of chitosan-graphene complex membrane could be clearly observed (Fig. 2g). And that may be attributed to the plentiful amino $\left(-\mathrm{NH}_{2}\right)$ and hydroxyl $(-\mathrm{OH})$ in the chitosan- graphene membrane contributing to the immobilization of CQDs (hydroxyl and carboxyl groups rich). Fig. 2h shows the EDS of synthesized CQDs obtained form the high-resolution TEM. In accordance with the results obtained from the XPS given in Fig. S5a, $\uparrow$ only carbon and oxygen element could be detected.

To investigate the surface property of modified electrode, electrochemical impedance spectroscopy (EIS) was implemented in 0.1 M phosphate buffer solution (PBS) containing $5 \mathrm{mM}\left[\mathrm{Fe}(\mathrm{CN})_{6}\right]^{3-/ 4-}$. As shown in Fig. 3, the electrochemical impedance (EIS) of the modified electrodes presents a semicircle at high frequencies (electron transfer resistance) and a linear part at low frequencies (solution diffusion resistance), simultaneously. ${ }^{31}$ The bare glassy carbon electrode had the lowest electron transfer resistance (Fig. 3a) with a smallest semicircle diameter among all the tested samples (Fig. 3b-d). The greatly increased semicircle diameter of chitosan membrane modified electrode (Fig. 3b) indicates the electron transfer between redox probe and electrode was impeded. Fig. $3 c$ and $d$ represent the electron transmission capacity of CG composite membrane and CQDs/CG composite, respectively, from which, a reduced diameter (less than the chitosan membrane modified electrode) could be detected, indicative the existence of CQDs can optimize the electron transfer capability.

The ECL experiment of the CQDs/CG composite modified electrode was carried out in $0.1 \mathrm{M}$ PBS containing $0.1 \mathrm{M}$ $\left(\mathrm{NH}_{4}\right)_{2} \mathrm{~S}_{2} \mathrm{O}_{8}$ and $0.1 \mathrm{M} \mathrm{KCl}$. And a cyclic voltammetry curve was recorded at the potential from 0 to $-1.4 \mathrm{~V}$ at the same time (Fig. 4). An obvious ECL emission could be detected, implying the successfully binding of CQDs with the chitosan-graphene membrane matrix.

Fig. 5 shows the ECL behaviors of different electrodes conducted in 0.1 M PBS solutions containing $0.1 \mathrm{M}\left(\mathrm{NH}_{4}\right)_{2} \mathrm{~S}_{2} \mathrm{O}_{8}$ and $0.1 \mathrm{M} \mathrm{KCl}$. Almost no obvious ECL signal could be detected for both the bare glassy carbon electrode (Fig. 5a) and chitosangraphene modified electrode (Fig. 5b). However, a distinct ECL peak could be observed for the CQDs/CG modified electrode (Fig. 5c). It demonstrates that the CQDs had been immobilized on the chitosan-graphene modified electrode and excellent photo-electricity properties could be maintained.

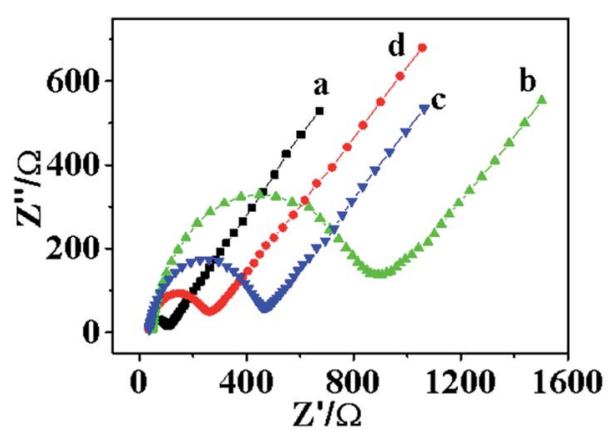

Fig. 3 Electrochemical impedance spectroscopy of bare or modified electrode: (a) EIS of bare glassy carbon electrode; (b) EIS of chitosan modified electrode; (c) EIS of chitosan-graphene modified electrode; (d) EIS of carbon quantum dots/chitosan-graphene modified electrode. 


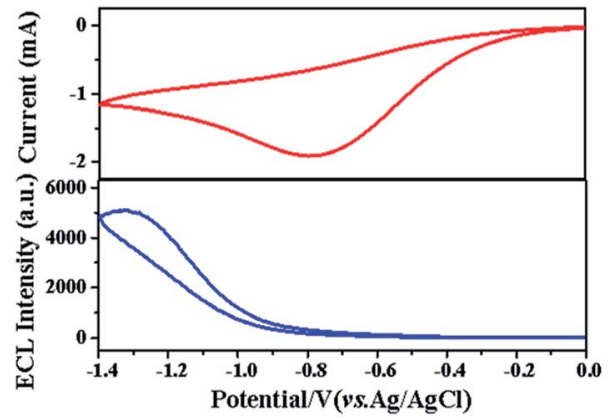

Fig. 4 Electrochemiluminescence and cyclic voltammetry behaviors of CQDs/chitosan-graphene composite modified electrode in $0.1 \mathrm{M}$ PBS.

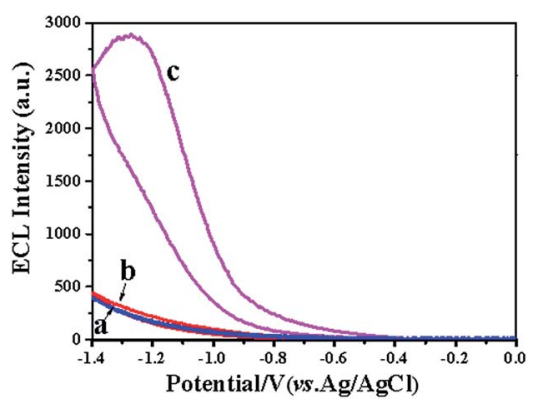

Fig. 5 ECL behaviors of bare or modified electrode: (a) ECL behavior of bare electrode, (b) ECL behavior of chitosan-graphene modified electrode; (c) ECL behavior of CQDs/chitosan-graphene modified electrode.

The ECL mechanism of CQDs in the presence of $\left(\mathrm{NH}_{4}\right)_{2} \mathrm{~S}_{2} \mathrm{O}_{8}$ might be as follows:

$$
\begin{gathered}
\mathrm{CQDs}+\mathrm{e}^{-} \rightarrow \mathrm{CQDs}^{\cdot-} \\
\mathrm{S}_{2} \mathrm{O}_{8}^{2-}+\mathrm{e}^{-} \rightarrow \mathrm{SO}_{4}{ }^{2-}+\mathrm{SO}_{4}{ }^{--} \\
\mathrm{CQDs}^{\cdot-}+\mathrm{SO}_{4}^{\cdot-} \rightarrow \mathrm{CQDs}^{*}+\mathrm{SO}_{4}{ }^{2-} \\
\mathrm{CQDs}^{*} \rightarrow \mathrm{CQDs}+h \gamma
\end{gathered}
$$

Previous reports have demonstrated that CQDs is a valuable ECL emitter. However, DA could act as a quencher preventing the reaction between CQDs and the coreactant $\left(\mathrm{NH}_{4}\right)_{2} \mathrm{~S}_{2} \mathrm{O}_{8} \cdot{ }^{32}$ Hence, the CQDs/CG composite electrode could be used for the detection of dopamine. The measurement was performed in $0.1 \mathrm{M}$ PBS containing $0.1 \mathrm{M}\left(\mathrm{NH}_{4}\right)_{2} \mathrm{~S}_{2} \mathrm{O}_{8}$ and $0.1 \mathrm{M} \mathrm{KCl}$. As delivered in Fig. 6, with the increase of dopamine concentration, the ECL intensity decreased continuously. A satisfying linear relationship between ECL signal and the concentration of dopamine $(0.06$ to $1.6 \mu \mathrm{M})$ was obtained. Furthermore, the detection limit could reach $0.028 \mu \mathrm{M}(\mathrm{S} / \mathrm{N}=3)$. The insert part in Fig. 6 shows the calibration curve of dopamine determination. The linear regression equation was $I=-6111.32 c(\mu \mathrm{M})+$ $7946\left(R^{2}=0.9582\right)$.

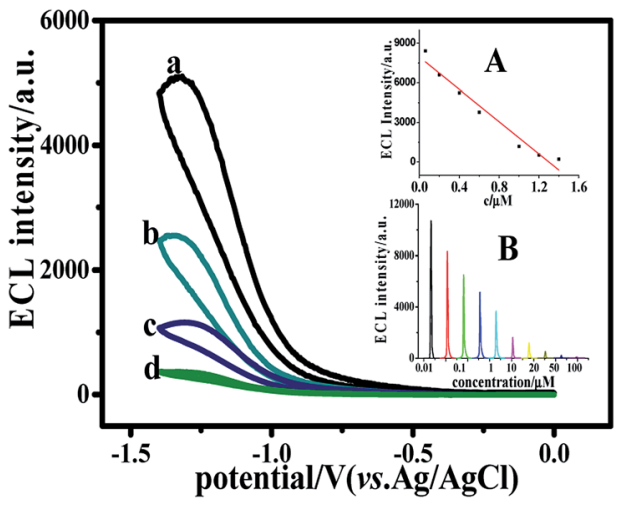

Fig. 6 Effect of dopamine concentration on ECL intensity of CQDs/ CG composite electrode in $0.1 \mathrm{M} \mathrm{PBS}$ containing $0.1 \mathrm{M}\left(\mathrm{NH}_{4}\right)_{2} \mathrm{~S}_{2} \mathrm{O}_{8}$ and $0.1 \mathrm{M} \mathrm{KCl}$ and (a) 0.5, (b) 0.9, (c) 1.1 and (d) $1.2 \mu \mathrm{M}$ dopamine at $50 \mathrm{mV}$ $\mathrm{s}^{-1}$. Inset: (A) calibration curve of dopamine detection; (B) ECL intensity change with the injection of dopamine.

A high selectivity is prerequisite for the real application of an electrode. Hence, an interference test was carried out in the solution contained $0.5 \mu \mathrm{M}$ DA together with the interfering substance, for example, ascorbic acid (AA), uric acid (UA), glucose, $\mathrm{K}^{+}$or $\mathrm{Na}^{+}$. And the concentration of the interfering particles are 100 times more concentrated than dopamine $\left(C_{\text {interference }}: C_{\mathrm{DA}}=100\right)$. According to previous report, ${ }^{33}$ as a cationic exchanger, chitosan has an excellent selectivity for DA. However, ascorbic acid (AA) and uric acid (UA) all carried a negative charge in neutral environment. Therefore, the CQDs/ chitosan-graphene film could hinder the reaching of ascorbic acid and uric acid to the electrode surface. As shown in Fig. 7, after the addition of ascorbic acid and uric acid, a decrease of about $12 \%$ and $10 \%$ could be detected for the AA and UA, respectively. While different with AA and UA, the interference of glucose, $\mathrm{Na}^{+}$and $\mathrm{K}^{+}$could be omitted. All these indicated the high-selectivity of the composite film for the DA.

To evaluate the stability of as-prepared CQDs, we measured the ECL behavior which had been kept refrigerated and protected from light for three months. The ECL intensity was remained approximately $100 \%$ after three months. Furthermore, the modified electrode was used once a week to detect the

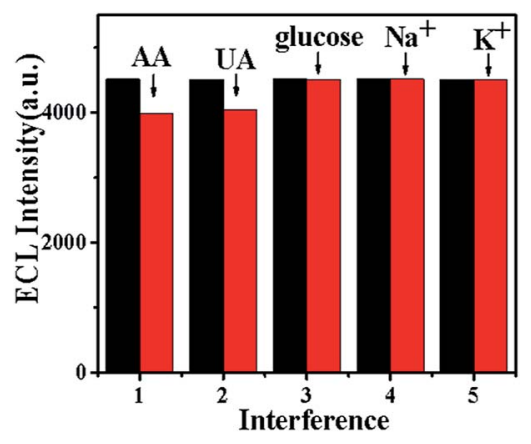

Fig. 7 Interference study of the CQDs/chitosan-graphene composite modified electrode towards glucose, $\mathrm{Na}^{+}, \mathrm{K}^{+}$, ascorbic acid (AA) and uric acid (UA) in $0.1 \mathrm{M}$ PBS containing $0.5 \mu \mathrm{M}$ dopamine. 


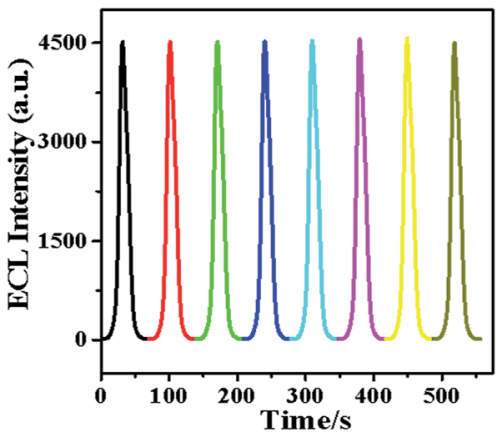

Fig. 8 The reproducibility of CQDs/chitosan-graphene composite modified electrode under continuous scanning for 8 circles in $0.1 \mathrm{M}$ PBS containing $0.5 \mu \mathrm{M}$ dopamine and $0.1 \mathrm{M} \mathrm{KCl}$.

Table 1 Detection of dopamine in serum samples

\begin{tabular}{lllll}
\hline Sample & Spiked $(\mu \mathrm{M})$ & Found $(\mu \mathrm{M})$ & $\begin{array}{l}\text { Recovery } \\
(\%)\end{array}$ & $\begin{array}{l}\text { RSD }(\%) \\
(n=3)\end{array}$ \\
\hline Serum 1 & 0.20 & 0.191 & 95.1 & 4.0 \\
Serum 2 & 0.40 & 0.386 & 96.5 & 3.1 \\
Serum 3 & 0.60 & 0.561 & 93.5 & 4.1
\end{tabular}

dopamine. Compared with the initial value, the intensity could be maintained at $80 \%$ after one month. All these validated the excellent stability of the as-prepared CQDs. Fig. 8 shows the reproducibility of the CQDs/chitosan-graphene composite modified electrode proceeded with a continuously potential scanning for 8 cycles. A stable ECL signal was received with a low relative-standard-deviation (RSD) of $0.6 \%$ after a long cycle of 8 times, further indicating the excellent stability of prepared composite sample.

The effective detection and quantitative analysis of the actual dopamine in human body fluids is of great significance to human health. The feasibility of the prepared CQDs/CG composite modified electrode for practical application was investigated by detecting the concentration of DA in human serum samples. These samples offered by student volunteers were centrifuged and the supernatant liquors were immediately used to the recoveries test without further treatment. Every sample was parallel tested three times and the results are shown in Table 1. As listed in Table 1, the recoveries and RSD $(n=3)$ were in the range of 93.5-96.5\% and 3.1-4.1\%, respectively. The obtained results demonstrated that the performance of prepared composite electrode was comparable to other reported methods and applicable for the determination of DA in real sample with adequate accuracy. The detail was given in the Table S2.†

\section{Conclusions}

We explore a new carbon source for preparation of CQDs using a POP derived porous carbon via nitric acid oxidation method. The POP was synthesized by mechanochemical Friedel-Crafts alkylation in solvent free conditions. Using the as-prepared
CQDs, we develop a simple and effective ECL detection method to DA based on CQDs/CG composite modified GCE. The complementary structure and synergistic function of CQDs and CG composite film endowed the composite electrode special properties. The ECL showed high selectivity to DA with an excellent interference resistance ability in a high concentration ratio of $100\left(C_{\text {interference }}: C_{\mathrm{DA}}=100\right)$. In addition, the sensor was applied for detection and quantitative analysis of the actual dopamine in human body fluids. And other novel porous organic polymer based precursors could be prepared for the preparation of CQDs with controllable structure and composition.

\section{Conflicts of interest}

There are no conflicts to declare.

\section{Acknowledgements}

This work was supported by the National Natural Science Foundation of China $(81774125,21705120)$, the Project of Shandong Province Higher Educational Science and Technology Program (J16LM04) and the Natural Science Foundation of Shandong Province (No. ZR2016HM47).

\section{Notes and references}

1 Q. Lu, X. Chen, D. Liu, C. Wu, M. Liu, H. Li, Y. Zhang and S. Yao, Talanta, 2018, 182, 428-432.

2 N. Mohseni and M. Bahram, Spectrochim. Acta, Part A, 2018, 193, 451.

3 M. Oh, E. Huh, M. S. Oh, J. S. Jeong and S. Li, J. Pharm. Biomed. Anal., 2018, 153, 110.

4 Y. Zhu, Z. Yang, M. Chi, M. Li, C. Wang and X. Lu, Talanta, 2018, 181, 431-439.

5 S. K. Shukla, A. Lavon, O. Shmulevich and H. B. Yoav, Talanta, 2018, 181, 57-64.

6 G. Xu, Z. A. Jarjes, V. Desprez, P. A. Kilmartin and J. T. Sejdic, Chemosensors, 2018, 107, 184-191.

7 M. Khan, X. Liu, Y. Tang, J. Zhu, W. Hu and X. Liu, Microchim. Acta, 2018, 185, 439.

8 A. J. Stewart, J. Hendry and L. Dennany, Anal. Chem., 2015, 87, 11847-11853.

9 X. Fu, X. Tan, R. Yuan and S. Chen, Biosens. Bioelectron., 2017, 90, 61-68.

10 L. Zhang, Y. Cheng, J. Lei, Y. Liu, Q. Hao and H. Ju, Anal. Chem., 2013, 85, 8001-8007.

11 Y. Dong, W. Tian, S. Ren, R. Dai, Y. Chi and G. Chen, Applied Materials \& Interfaces, 2014, 6, 1646.

12 M. M. Richter, Chem. Rev., 2004, 104, 3003-3036.

13 E. Han, L. Ding, H. Lian and H. Ju, Chem. Commun., 2010, 46, 5446-5448.

14 S. E. K. Kirschbaum and A. J. Baeumner, Anal. Bioanal. Chem., 2015, 407, 3911-3926.

15 S. Liu, X. Zhang, Y. Yu and G. Zou, Anal. Chem., 2014, 86, 2784-2788. 
16 (a) A. J. Stewart, E. J. Reilly, R. D. Moriarty, P. Bertoncello, T. E. Keyes, R. J. Forster and L. Dennany, Electrochim. Acta, 2015, 157, 8-14; (b) M. Zhang, T. Zhao, J. Dou, Z. Xu, W. Zhang, X. Chen, X. Wang and B. Zhou, ChemElectroChem, 2019, DOI: 10.1002/celc.201901586.

17 X. Li, S. Q. Yu, T. Yan, Y. Zhang, B. Du, D. Wu and Q. Wei, Biosens. Bioelectron., 2017, 89, 1020-1025.

18 G. Zhang, S. Deng, X. Zhang and D. Shan, Electrochim. Acta, 2016, 190, 64-68.

19 Q. Zhai, H. Xing, X. Zhang, J. Li and E. Wang, Anal. Chem., 2017, 89, 7788-7794.

20 D. M. Hercules, Science, 1964, 145, 808-809.

21 Y. Yan, Q. Liu, X. Dong, N. Hao, S. Chen, T. You, H. Mao and K. Wang, Microchim. Acta, 2016, 183, 1591-1599.

22 R. M. Shereema, V. Sankar, K. G. Raghu, T. Rao and S. Shankar, Electrochim. Acta, 2015, 182, 588.

23 C. Xiong, W. Liang, H. Wang, Y. Zheng, Y. Zhuo, Y. Chai and R. Yuan, Chem. Commun., 2016, 52, 5589-5592.

24 R. Wang, K. Lu, Z. Tang and Y. X. Zhang, J. Mater. Chem. A, 2017, 5, 3717.

25 P. Namdaria, B. Negahdarib and A. Eatemadib, Biomed. Pharmacother., 2017, 87209-87222.
26 B. Zhou and L. Chen, Acta Chim. Sin., 2015, 73, 487-497.

27 G. Liu, C. Ma, B. K. Jin, Z. Chen and J. Zhu, Anal. Chem., 2018, 90, 4801.

28 (a) X. J. Song, J. Fu, J. Wang, C. Y. Li and Z. H. Liu, Microchim. Acta, 2018, 185, 369; (b) B. Zhou, F. Yan, X. Li, J. Zhou and W. Zhang, ChemSusChem, 2019, 12, 915-923; (c) B. Zhou, L. Liu, Z. Yang, X. Li, Z. Wen and L. Chen, ChemElectroChem, 2019, 6, 485-492; (d) B. Zhou, L. Liu, P. Cai, G. Zeng, X. Li, Z. Wen and L. Chen, J. Mater. Chem. A, 2017, 5, 22163-22169; (e) B. Zhou, X. Hu, G. Zeng, S. Li, Z. Wen and L. Chen, ChemSusChem, 2017, 10, 2955-2961.

29 S. Kulchat, W. Boonta, A. Todee, P. Sianglam and W. Ngeontae, Spectrochim. Acta, Part A, 2018, 196, 7.

30 J. Zhou, T. Q. Han, H. M. Ma, T. Yan, X. H. Pang, Y. Y. Li and Q. Wei, Anal. Chim. Acta, 2015, 889, 82-89.

31 T. Wang, S. Y. Zhang, C. Mao, J. Song, H. Niu, B. Jin and Y. Tian, Biosens. Bioelectron., 2012, 31, 369-375.

32 F. Sun, F. Chen, W. Fei, L. Sun and Y. Wu, Sens. Actuators, B, 2012, 702, 166-167.

33 Q. Huang, S. Hu, H. Zhang, J. Chen, Y. He, F. Li, W. Weng, J. Ni, X. Bao and Y. Lin, Analyst, 2013, 138, 5417-5423. 